\title{
Chronic sclerosing sialadenitis of the sublingual gland: case report and literature review
}

\author{
Filippos Thomopoulos ${ }^{1}$, Nikolaos G. Nikitakis², Argyrios Daskalopoulos ${ }^{2}$, Evangelia Piperi², Nikolaos \\ Kolomvos $^{3}$, Alexandra Sklavounou ${ }^{2}$ \\ 'Undergraduate student, School of Dentistry, National and Kapodistrian University of Athens, Athens 11527, Greece. \\ ${ }^{2}$ Department of Oral Medicine and Pathology, School of Dentistry, National and Kapodistrian University of Athens, Athens 11527, \\ Greece. \\ ${ }^{3}$ Department of Oral and Maxillofacial Surgery, School of Dentistry, National and Kapodistrian University of Athens, Athens 11527, \\ Greece.
}

Correspondence to: Dr. Argyrios Daskalopoulos, Department of Oral Medicine and Pathology, School of Dentistry, National and Kapodistrian University of Athens, 2 Thivon St., Athens 11527, Greece. E-mail: adaskal@hotmail.gr

How to cite this article: Thomopoulos F, Nikitakis NG, Daskalopoulos A, Piperi E, Kolomvos N, Sklavounou A. Chronic sclerosing sialadenitis of the sublingual gland: case report and literature review. Stomatological Dis Sci2018;2:8.

http://dx.doi.org/10.20517/2573-0002.2017.22

Received: 23 Dec 2017 First Decision: 4 Apr 2018 Revised: 14 May 2018 Accepted: 18 May 2018 Published: 19 Jul 2018

Science Editor: Athanasios Poulopoulos Copy Editor: Jun-Yao Li Production Editor: Cai-Hong Wang

\begin{abstract}
Chronic sclerosing sialadenitis (CSS) is a chronic inflammatory condition of uncertain etiology, primarily affecting the submandibular glands. It has recently been proposed that CSS belongs to the immunoglobulin G4 (IgG4)-related disease spectrum, although the exact relationship between these two entities requires further elucidation. Our aim is to present a case of bilateral CSS of the sublingual glands and discuss the possible underlying pathogenetic mechanisms. A 66-year-old woman presented with a 7-month history of an asymptomatic firm swelling of the floor of the mouth. Clinically, a diffuse, multinodular swelling of elastic to firm consistency was observed across the sublingual folds bilaterally. An incisional biopsy revealed a lymphoplasmacytic infiltrate with lymphoid follicle formation, along with acinar degeneration, fibrosclerosis and ductal dilation. Immunohistochemical investigation ruled out lymphoproliferative diseases revealing a polyclonal lymphoplasmacytic population with only a few scattered IgG4-positive plasma cells. IgG4 serum levels were also normal. No treatment was administered with progressive spontaneous resolution of the swelling and no evidence of recurrence at 2 years follow-up. CSS is a benign salivary gland disorder, which, at least in a subset of cases, may represent an IgG4-associated disorder. Therefore, immunohistochemical evaluation of the $\lg G 4 / \lg G$ plasma cell ratio and the $\lg G 4$ serum levels is a recommended investigation in the diagnostic work-up of CSS cases.
\end{abstract}

Keywords: Chronic sclerosing sialadenitis, immunoglobulin G4-related sialadenitis, sialolithiasis, sublingual salivary gland

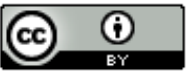

(C) The Author(s) 2018. Open Access This article is licensed under a Creative Commons Attribution 4.0 International License (https://creativecommons.org/licenses/by/4.0/), which permits unrestricted use, sharing, adaptation, distribution and reproduction in any medium or format, for any purpose, even commercially, as long as you give appropriate credit to the original author(s) and the source, provide a link to the Creative Commons license, and indicate if changes were made. 


\section{INTRODUCTION}

Chronic sclerosing sialadenitis (CSS) is a chronic fibrosing inflammatory condition, first described by Küttner $^{[1]}$ in 1896, most commonly affecting the submandibular glands ${ }^{[2-4]}$. Clinically, CSS may present as a unilateral or rarely bilateral, asymptomatic or mildly symptomatic firm swelling of the involved gland, often mimicking a neoplasm ${ }^{[2,3,5]}$. Histologically, CSS shows marked lymphocytic infiltration with reactive lymphoid follicle formation, periductal fibrosis, acinar atrophy and interlobular sclerosis ${ }^{[3,5,6]}$.

Although the pathogenesis of CSS remains uncertain, a wide range of etiologic factors has been proposed including sialolithiasis, secretory disorders, infectious agents, ductal abnormalities and immunopathologic reactions ${ }^{[2,5]}$. Kitagawa et al. ${ }^{[7]}$ suggested that CSS belongs to the immunoglobulin G4-related disease (IgG4RD) spectrum, a recently described, systemic, immune mediated disease with multi-organ involvement. Since then, the possible association between chronic sclerosing sialadenitis and IgG4-RD has been postulated.

Here we report a very rare case of bilateral CCS of the sublingual glands in a 66-year-old woman. The clinical, microscopic and immunopathological features are presented and the possible underlying pathogenetic mechanisms of this, possibly under-recognized, condition are also discussed.

\section{CASE REPORT}

A 66-year-old female presented to the Oral Medicine Clinic, School of Dentistry, National and Kapodistrian University of Athens, Greece for evaluation of a mildly painful swelling of the floor of the mouth of 2 weeks duration. At the onset, the patient visited the Oral and Maxillofacial Surgery Clinic of a local hospital, where broad-spectrum antibiotics (amoxicillin $1 \mathrm{~g}$ bid plus metronidazole $500 \mathrm{mg}$ tid) were administered for 1 week with only minimal improvement of the swelling.

According to the patient, the first episode of swelling at the area was observed approximately 7 months ago. At that time, panoramic radiograph, computed tomography (CT) scan and ultrasound (US) imaging were performed without remarkable pathologic findings. However, imaging of the sublingual gland region with CT scan was distorted due to the metal artifacts produced by the patient's dental prosthetic appliances. Antibiotic treatment was prescribed and the swelling subsided within 1 week.

The medical history was remarkable for a papillary thyroid carcinoma, developing in the context of Hashimoto's thyroiditis, diagnosed 3 years ago and managed with thyroidectomy without radioactive iodine treatment. Since then, the patient was on levothyroxine $75 \mathrm{mg}$ qd. Moreover, she had hypertension treated with a combination of an angiotensin II receptor antagonist and a thiazide diuretic (olmesartan medoxomil $20 \mathrm{mg}$ plus hydrochlorothiazide $12.5 \mathrm{mg} \mathrm{qd}$ ) for the last year and hypercholesterolemia, for which she was receiving atorvastatin $(20 \mathrm{mg}$ qd) for the last 7 months. She also reported cardiac arrhythmia managed with a non-selective competitive beta-adrenergic receptor blocker I (class III antiarythmic, sotalol hydrochloride $80 \mathrm{mg}$ tabs qd) for the last 2 years. A recent complete blood cell count showed mild anemia and an elevated erythrocyte sedimentation rate (ESR).

Clinically, a diffuse, firm, non-tender on palpation swelling was noticed across the sublingual folds bilaterally, more prominently on the left side [Figure 1]. Saliva production from all major salivary glands and unstimulated whole saliva flow rate at 5 min were within normal limits. The remaining of the oral mucosa was also normal, no dental pathology in the lower teeth was identified and no cervical lymphadenopathy was noted. The differential diagnosis included chronic sialadenitis, salivary gland neoplasms, granulomatous disorders (e.g., sarcoidosis), Sjogren's syndrome (although the salivary flow rate was normal) and deposition diseases, such as amyloidosis. 


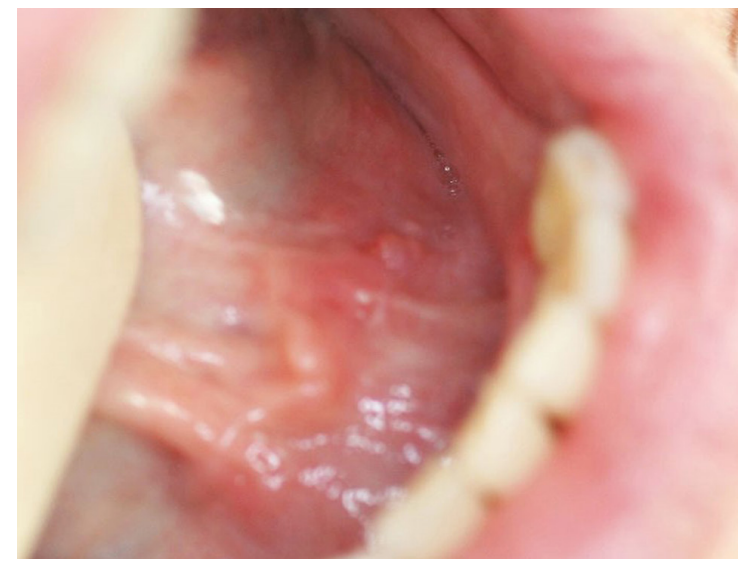

Figure 1. Diffuse, firm, palpable swelling across the sublingual folds bilaterally, more prominently on the left

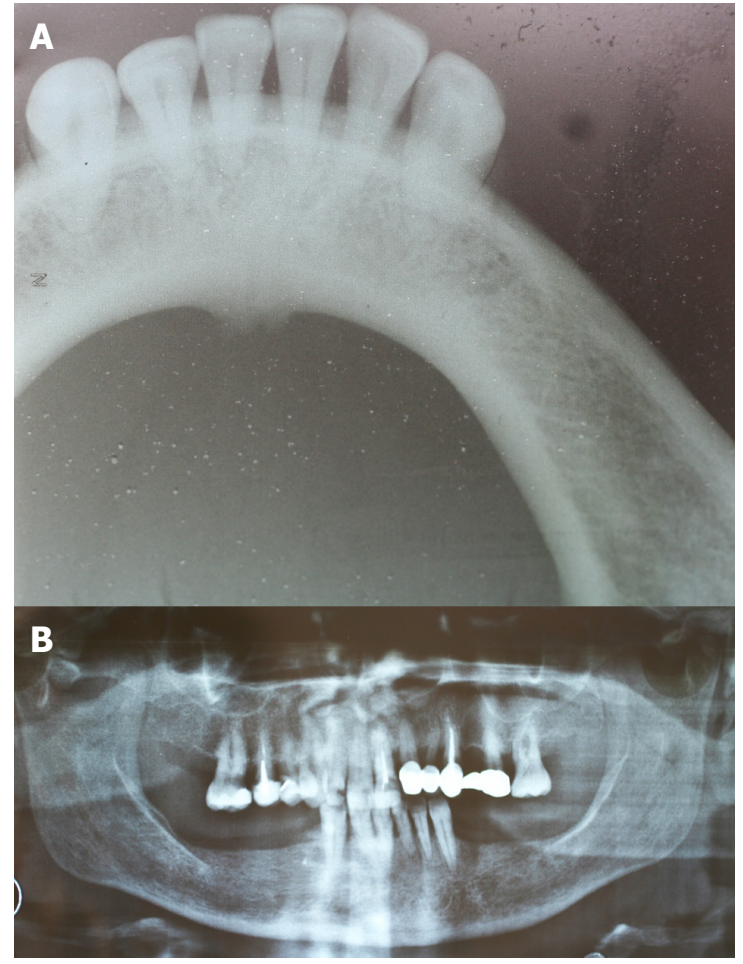

Figure 2. Occlusal (A) and panoramic (B) radiographs did not reveal any pathologic findings

Occlusal and panoramic radiographs were non-contributory [Figure 2], while a new US and magnetic resonance imaging [Figure 3] of the sublingual/submandibular region did not reveal any evidence of a spaceoccupying or other lesion.

An incisional biopsy of the left sublingual gland swelling was performed under topical anesthesia. Histopathologic examination revealed a dense diffuse lymphoplasmacytic infiltration of the salivary gland parenchyma with prominent lymphoid follicle formation and preservation of lobular architecture, extensive acinar degeneration, ductal dilation and dense interlobular fibrosis [Figure 4]. Although several serial sections were examined, no signs of sialolith (as a possible cause of obstructive sialadenitis) were noted. Further, the lymphoid cells did not demonstrate atypia and immunohistochemical investigation revealed a polyclonal 


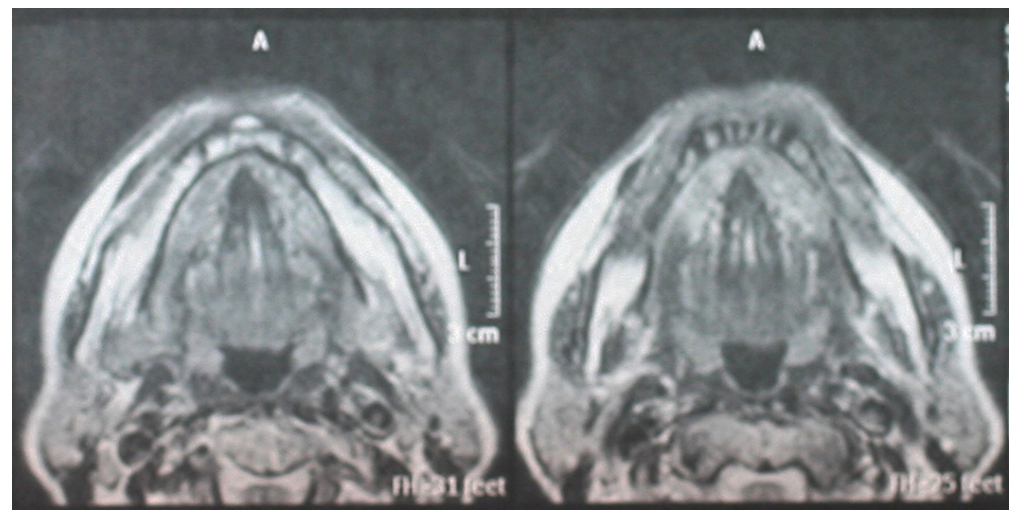

Figure 3. Magnetic resonance imaging did not reveal evidence of a space occupying or other lesion in the sublingual-submandibular area

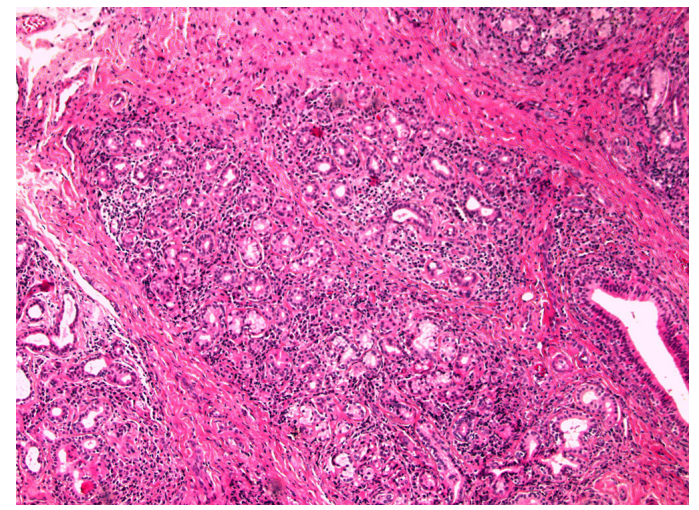

Figure 4. Photomicrograph of sublingual gland biopsy showing diffuse lymphoplasmacytic infiltration with acinar degeneration, dense fibrosis and ductal dilation in the absence of sialolith formation (HE, 200×)

lymphoplasmacytic population, ruling out a lymphoproliferative disorder. Moreover, lymphoepithelial lesions (suggestive of Sjögren's syndrome) were absent.

Based on the clinical and microscopic findings, a provisional diagnosis of CSS was rendered. To exclude the possibility of an IgG4-related disease, further immunohistochemical studies were performed, showing only a few scattered IgG4 positive plasma cells with a low IgG4/IgG ratio. In addition, serological examination, including IgG subtypes, was normal with the exception of an elevated IgG2 level [Table 1].

The post-operative course was uneventful with complete resolution of symptoms and a significant decrease of the swelling within 2 weeks. The residual swelling was spontaneously resolved in the course of the next few weeks. During regular follow up examination for the next 2 years, no signs of recurrences were noticed; only a minimal asymptomatic firmness was palpable along the left sublingual fold, possibly due to scar tissue from the previous biopsy procedure.

\section{DISCUSSION}

CSS usually affects middle aged or elderly patients with a slight male predominance ${ }^{[8-10]}$. Clinically, it may present as a unilateral or rarely bilateral, asymptomatic or mildly painful, firm swelling of the involved salivary gland $^{[2,3,5]}$. Salivary flow rate is usually normal or slightly decreased ${ }^{[8]}$. CSS typically affects the submandibular gland; however, involvement of the parotid, the sublingual gland and the minor salivary glands has also been reported $^{[3,11,12]}$. To the best of our knowledge, CSS affecting the sublingual gland is exceedingly rare ${ }^{[12]}$. 
Table 1. Results of serological examination

\begin{tabular}{lll}
\hline Test & Results & Normal rates \\
\hline ANA & Negative & Negative $<1 / 40$ \\
Anti-Ro, anti-La & Negative & Negative $<0.8$ \\
RF & $12 \mathrm{IU} / \mathrm{mL}$ & $0-30 \mathrm{IU} / \mathrm{mL}$ \\
CRP & $1.8 \mathrm{mg} / \mathrm{L}$ & $<5 \mathrm{mg} / \mathrm{L}$ \\
$\operatorname{lgA}$ & $250 \mathrm{mg} / \mathrm{dL}$ & $72-400 \mathrm{mg} / \mathrm{dL}$ \\
$\operatorname{lgM}$ & $161 \mathrm{mg} / \mathrm{dL}$ & $40-235 \mathrm{mg} / \mathrm{dL}$ \\
$\operatorname{lgG}$ & $1450 \mathrm{mg} / \mathrm{dL}$ & $490-1140 \mathrm{mg} / \mathrm{dL}$ \\
$\operatorname{lgG1}$ & $805 \mathrm{mg} / \mathrm{dL}$ & $490-1140 \mathrm{mg} / \mathrm{dL}$ \\
$\operatorname{lgG2}$ & $549 \mathrm{mg} / \mathrm{dL}$ & $150-460 \mathrm{mg} / \mathrm{dL}$ \\
$\operatorname{lgG3}$ & $54.1 \mathrm{mg} / \mathrm{dL}$ & $20-110 \mathrm{mg} / \mathrm{dL}$ \\
$\operatorname{lgG4}$ & $68.2 \mathrm{mg} / \mathrm{dL}$ & $8-140 \mathrm{mg} / \mathrm{dL}$ \\
\hline
\end{tabular}

Histopathologically, the lesions of CSS, similar to our case, show marked lymphoplasmacytic infiltration with formation of reactive lymphoid follicles, extensive fibrosis, ductal dilatation, acinar degeneration and, in some cases, obliterative phlebitis ${ }^{[8,13]}$. Seifert and Donath ${ }^{[14]}$ in 1977 proposed four histological stages of progressive inflammation in CSS. At the initial stage, lymphocytic infiltration around dilated ducts containing inspissated material is observed. Stage 2 is characterized by an increased lymphocytic infiltration with formation of lymphoid follicles, interstitial fibrosis, acinar atrophy and inspissated secretions in the ductal system, while ductal epithelium may show focal metaplasia with proliferation. At stage 3, the lymphocytic infiltration is even more intense, accompanied by lymphoid follicle formation, parenchymal atrophy, periductal hyalinization and sclerosis, while ductal epithelium metaplasia may also be present. At the last stage, inflammation leads to the destruction of the gland with parenchymal loss and sclerosis ${ }^{[14]}$. The microscopic findings of our case were more consistent with stage 2 .

The histopathologic differential diagnosis of CSS includes mainly Sjögren's syndrome and malignant lymphomas. Features such as presence of dense fibrosis, absence of lymphoepithelial lesions and infiltration by abundant plasma cells help distinguish CSS from Sjögren's syndrome ${ }^{[8]}$. CSS must also be differentiated from malignant lymphomas, especially non-Hodgkin B-cell lymphomas, which, albeit rare, represent the most common type of lymphoma affecting the salivary glands. In contrast to lymphomas, cellular atypia in CSS is absent and B-cell polyclonality is revealed immunohistochemically, as shown in our case. On the other hand, a rare case of extranodal marginal zone B-cell lymphoma of MALT type arising on the grounds of CSS has been described and, as such, regular follow-up until complete resolution of CSS may be indicated ${ }^{[15]}$.

The pathogenesis of CSS remains obscure. Initially, sialoliths were considered the most likely etiologic factor; it has been reported that, approximately $29 \%$ up to $83 \%$ of CSS cases, are related to the presence of sialoliths ${ }^{[4,10,1]}$. In cases of chronic obstructive sialadenitis, the microscopic appearance is characterized by mild interlobular fibrosis with lack of the characteristic lymphoid follicle formation and intense lymphoplasmacytic infiltration ${ }^{[7,8]}$. However, it remains unclear whether the development of sialoliths is the cause or the result of the inflammatory process ${ }^{[4,11]}$. In addition, in $70 \%$ of cases in which a sialolith was present, surgical excision did not result in resolution of the swelling ${ }^{[3]}$. Moreover, the progressive character of the inflammation observed in CSS, cannot be explained solely by the obstructive process ${ }^{[5]}$. Hence, it appears more likely that sialolithiasis is a secondary process to sialadenitis that may not be implicated in the pathogenesis of $\operatorname{CSS}^{[11,16]}$.

Although our patient was treated with a thiazide diuretic drug that may cause decreased salivary flow and dehydration predisposing to chronic obstructive sialadenitis, serial histopathologic sections did not reveal any findings suggestive of either inspissated material or sialolith formation. Further, normal salivary flow rate from all major salivary glands was noted. 


\section{Criteria}

1. Clinical examination shows characteristic diffuse/localized swelling or masses in single or multiple organs

2. Hematological examination shows elevated serum IgG4 concentrations ( $\geq 135 \mathrm{mg} / \mathrm{dL})$

3. Histopathologic examination shows:

(1) marked lymphocyte and plasmacyte infiltration and fibrosis;

(2) infiltration of IgG4-positive plasma cells: ratio of IgG4/IgG positive plasma cells > 40\% and > 10 IgG4-positive plasma cells/HPF Definite: $1+2+3$

Probable: $1+3$

Possible: $1+2$

Other authors suggest that in patients with CSS (or Küttner's tumor), a possible underlying secretory disorder of the affected gland may lead to the presence of inspissated saliva in the small ducts and subsequent chronic inflammation. This type of inflammation is called obstructive electrolyte sialadenitis ${ }^{[5,7,17]}$. In our patient, although sialectasia was present, no inspissated material was observed histopathologically in the ductal system, while unstimulated whole saliva rate was normal. On the other hand, the histopathological appearance of CSS lesions characterized by the presence of dense lymphocytic infiltration with lymphoid follicle formation and destruction of the gland, suggests that immunopathological reactions may be responsible for $\mathrm{CSS}^{[5]}$.

Kitagawa et al. ${ }^{[7]}$ in 2005 gave a new insight into the pathogenesis of CSS suggesting that it belongs to the IgG4related disease (IgG4-RD) spectrum, a recently recognized systemic disease with multi-organ involvement. IgG4-RD is a distinct condition characterized by diffuse enlargement or nodular/hyperplastic lesions of the involved organs, due to a prominent infiltration by lymphocytes and abundant IgG4-positive plasma cells, accompanied by a variable degree of fibrosis. Increased serum IgG4 levels and a good responsiveness to corticosteroids are also observed. Patients with IgG4-RD may have, either simultaneously or subsequently, multi-organ lesions or only single organ involvement ${ }^{[7,13]}$. This entity came into light first by Hamano et al. ${ }^{[18]}$ in 2001, who reported that the high serum concentrations of IgG4 in patients with sclerosing pancreatitis were reduced effectively with corticosteroid treatment, resulting in clinical remission. In Table 2, the diagnostic criteria proposed by Okazaki and Umehara ${ }^{[13]}$ in 2012 for IgG4-RD have been summarized.

The systemic nature of IgG4-RD was revealed by later studies that reported the involvement of numerous organs, including the lacrimal/salivary glands, thyroid gland, bile ducts, lung, kidney, retroperitoneum, central nervous system, liver, lymph nodes, prostate gland, skin and arteries. In Table 3, the IgG4-related disease spectrum is summarized ${ }^{[13]}$. As a result, over the last decade, the spectrum of IgG4-RD has been broadened comprising a variety of previously described diseases that seemed to be unrelated, such as Mikulicz's disease (MD), Küttner's tumor, autoimmune pancreatitis, inflammatory pseudotumor of the orbit, retroperitoneal fibrosis, Riedel thyroiditis and a subtype of Hashimoto thyroiditis $(\mathrm{HT})^{[19,20]}$.

$\mathrm{MD}$, formerly considered to be a part of Sjögren's syndrome (SS) clinical spectrum, is characterized by the persistent symmetrical swelling of at least two lacrimal and major salivary glands for more than three month ${ }^{[8]}$. Recent studies have revealed that SS and MD are two distinct entities, the latter exhibiting the characteristic features of IgG4-RD ${ }^{[17,21]}$. In addition, Küttner's tumor and MD both show salivary gland involvement with similar histopathological features, but CSS lesions exhibit more intense fibrosis ${ }^{[8,19]}$. In contrast, other authors suggest that CSS and MD may actually represent two variants of IgG4-associated sialadenitis ${ }^{[8]}$.

Although IgG4-RD is a well recognized disease, its pathogenesis and the role of IgG4 remain unclear. Autoimmunity has been speculated as the most probable pathogenetic mechanism. Lactoferrin and carbonic anhydrase, expressed in exocrine organs such as salivary gland and pancreas are considered potential autoantigens. Although autoantibodies against these antigens are the most often detectable ones in the serum of IgG4-RD patients, autoantibodies against the IgG4 molecule have not been detected ${ }^{[19,22]}$. It still remains 
Table 3. IgG4-related disease spectrum (modified from Okazaki and Umehara ${ }^{[13]}$, 2012)

\begin{tabular}{l}
\hline Spectrum \\
\hline Autoimmune pancreatitis type 1(AIP) \\
IgG4-related dacryoadenitis \\
IgG4-related sialadenitis \\
IgG4-related sclerosing cholangiitis \\
IgG4-related kidney disease \\
IgG4-related retroperitoneal fibrosis \\
IgG4-related thyroiditis \\
IgG4-related lypmhadenopathy \\
IgG4-related pulmonary disease \\
IgG4-related prostatitis \\
IgG4-related gastrointestinal disease \\
IgG4-related skin disease \\
IgG4-related aortitis \\
IgG4-related pachymeningitis \\
IgG4-related hypophysitis
\end{tabular}

unclear whether IgG4 is implicated directly or is just a bystander in the course of pathogenesis of IgG4-RD, during which an unknown primary inflammatory process leads to a secondary IgG4 over expression ${ }^{[2,23]}$.

Recent studies have shown that IgG4-RD is characterized by the predominant activation of T-helper type 2 (Th2) and regulatory T-lymphocytes (Treg cells) ${ }^{[1,22-24]}$. The Th2 cells produce interleukin (IL)-4, IL-5, IL-10, IL13,while Treg cells produce IL-10 and transforming growth factor-beta (TGF- $\beta)^{[19,22]}$. IL-10 has the ability to direct $\mathrm{B}$ cells to produce IgG4 and, as a result, to induce an IgG4 class switch, while TGF- $\beta$ induces fibroplasias ${ }^{[1,22-24]}$.

At present, there is an increasing evidence that CSS is an IgG4-RD ${ }^{[8,9,19]}$. Although our case did not meet the serological and histopathological diagnostic criteria for IgG4-related disease, as proposed by Okazaki and Umehara ${ }^{[13]}$ in 2012 [Table 2], a rare case of IgG4-RD-like multi-organ condition with involvement of the salivary glands, absence of IgG4 plasma cell infiltration and normal serum IgG4 levels has been reported recently by Hara et al. ${ }^{[23]}$ in 2014 . The authors hypothesized that a possible abnormality of the IL-10 signaling might have not allowed the IgG4 class switch.

Another aspect of IgG4-RD that has attracted a strong attention recently is the relationship between IgG4 and malignancy. Sporadic cases of tumors occurring in a background of IgG4-RD have been reported. Ito et al.$^{[25]}$ in 2011 described a case of thyroid papillary carcinoma accompanied by HT in a patient with IgG4-RD disease that involved the salivary glands, the pancreas and the biliary tract. They also reported that IgG4 positive plasma cells and fibrosis surrounded solid tumor nests, both at the primary tumor site and the metastatic lymph nodes ${ }^{[25,26]}$. As mentioned before, a subtype of HT is considered to be IgG4-related ${ }^{[20]}$. Moreover, HT is considered a risk factor for papillary thyroid carcinoma. Taşli et al.$^{[27]}$ in 2014 investigated the possible role of IgG4 in the relationship between these two entities and reported an increased papillary carcinoma prevalence in IgG4 positive HT cases, compared to the IgG4 negative ones, suggesting that IgG4 plasma cells may be implicated in carcinogenesis ${ }^{[27]}$. Interestingly, our patient had a history of thyroidectomy due to a papillary thyroid carcinoma on the grounds of HT 3 years before the diagnosis of CSS, although the relationship between IgG4 positive levels and the underlying thyroiditis had not been investigated.

Concerning the treatment of CSS, there is a lack of specific protocols in the literature. However, the treatment options that have been reported so far vary between conservative to more aggressive. In cases of CSS due to sialolithiasis, surgery is the treatment of choice ${ }^{[2]}$. Spontaneous regression of the lesions in the absence of sialolithiasis has also been reported ${ }^{[3]}$. Since an IgG4-related process has been considered to be implicated in the CSS pathogenesis and a favorable response to corticosteroid treatment has been observed in IgG4-RD cases, this treatment option has also been applied with good results ${ }^{[2,28-30]}$. 
In conclusion, CCS is an under-recognized benign salivary gland disorder of unknown etiology that may represent, at least in some cases, an IgG4-associated disease ${ }^{[30,31]}$. In this regard, immunohistochemical evaluation of the IgG4/IgG positive plasma cell ratio and the IgG4 serum levels should be performed in all cases although histopathologic examination remains the main diagnostic procedure ${ }^{[32]}$. Although our case did not meet the immunohistochemical and serological diagnostic criteria of IgG4-related sialadenitis, a rare case of IgG4-RD-like lesions with normal serum and scanty IgG4 tissue positivity has been described. Additionally, no sialoliths were observed nor any other cause could be identified. Therefore, long term follow-up in all CSS cases, even in the absence of typical IgG4-RD features, is recommended.

\section{DECLARATIONS}

\section{Acknowledgments}

The authors would like to thank Dr. Dimitra Rondogianni, Head, Department of Pathology, Evaggelismos General Hospital, Athens, for her expert opinion.

\section{Authors' contributions}

Case report concepts: Nikitakis NG, Sklavounou A

Diagnosis and management: Nikitakis NG, Daskalopoulos A

Quality control of data: Piperi E, Sklavounou A

Data analysis and interpretation: Thomopoulos F, Nikitakis NG, Piperi E, Sklavounou A

Literature review: Thomopoulos F, Daskalopoulos A, Kolomvos N

Manuscript preparation: Thomopoulos F, Nikitakis NG, Daskalopoulos A, Piperi E

Manuscript editing: Nikitakis NG, Daskalopoulos A, Piperi E, Kolomvos N

\section{Availability of data and materials}

Not applicable.

\section{Financial support and sponsorship}

None.

\section{Conflicts of interest}

The authors declare that there are no conflicts of interest related to this study.

\section{Ethical approval and consent to participate}

The patient's consent was provided to us. The study was approved by the Review Board of the affiliated University while no ethical issues were considered during the study.

\section{Consent for publication}

Not applicable.

\section{Copyright}

(c) The Author(s) 2018.

\section{REFERENCES}

1. Küttner H. Über entzündliche tumoren der submaxillar-speicheldrüse. Bruns 'Beitr Klin Chir 1896;15:815-28. (in German)

2. Pandarakalam C, Goebel WM, Seyer B. Chronic sclerosing sialadenitis or Küttner's tumor associated with a giant sialolith: a case report. Oral Surg Oral Med Oral Pathol Oral Radiol 2013;115:e38-40.

3. Williams HK, Connor R, Edmondson H. Chronic sclerosing sialadenitis of the submandibular and parotid glands: a report of a case and review of the literature. Oral Surg Oral Med Oral Pathol Oral Radiol 2000;89:720-3.

4. de Vicente JC, López-Arranz E, García J, López-Arranz JS. Chronic sclerosing sialadenitis of the parotid gland. Oral Surg Oral Med 
Oral Pathol Oral Radiol 2003;96:77-80.

5. Tiemann M, Teymoortash A, Schrader C, Werner JA, Parwaresch R, Seifert G, Klöppel G. Chronic sclerosing sialadenitis of the submandibular gland is mainly due to a T lymphocyte immune reaction. Mod Pathol 2002;15:845-52.

6. Neville BW, Damm DD, Allen CM, Bouquot JE. Oral and Maxillofacial Pathology. 3rd ed. St. Louis: Saunders, Elsevier; 2009. p. 461-2.

7. Kitagawa S, Zen Y, Harada K, Sasaki M, Sato Y, Minato H, Watanabe K, Kurumaya H, Katayanagi K, Masuda S, Niwa H, Tsuneyama K, Saito K, Haratake J, Takagawa K, Nakanuma Y. Abundant IgG4-positive plasma cell infiltration characterizes chronic sclerosing sialadenitis (Küttner's tumor). Am J Surg Pathol 2005;29:783-91.

8. Geyer JT, Deshpande V. IgG4-associated sialadenitis. Curr Opin Rheumatol 2011;23:95-101.

9. Laco J, Ryska A, Celakovsky P, Dolezalova H, Mottl R, Tucek L. Chronic sclerosing sialadenitis as one of the immunoglobulin G4related diseases: a clinicopathological study of six cases from Central Europe. Histopathol 2011;58:1157-63.

10. Ellis GL. Lymphoid lesions of salivary glands: malignant and benign. Med Oral Patol Oral Cir Bucal 2007;12:E479-85.

11. Blanco M, Mesko T, Cura M, Cabello-Inchausti B. Chronic sclerosing sialadenitis (Küttner's tumor): unusual presentation with bilateral involvement of major and minor salivary glands. Ann Diagn Pathol 2003;7:25-30.

12. Flaitz CM. Chronic sclerosing sialadenitis of the sublingual gland. Am J Dent 2001;14:335-36.

13. Okazaki K, Umehara H. Are classification criteria for IgG4-RD now possible? The concept of IgG4-related disease and proposal of comprehensive diagnostic criteria in Japan. Int J Rheumatol 2012;2012:357071.

14. Seifert G, Donath K. On the pathogenesis of the Küttner tumor of the submandibular gland. Analysis of 349 cases with chronic sialadenitis of the submandibular. HNO 1977;25:81-92.

15. Ochoa ER, Harris NL, Pilch BZ. Marginal zone B-cell lymphoma of the salivary gland arising in chronic sclerosing sialadenitis (Küttner tumor). Am J Surg Pathol 2001;25:1546-50.

16. Harrison JD, Epivatianos A. Immunoprofile of Küttner tumor (chronic sclerosing sialadenitis). Int J Surg Pathol 2010;18:443.

17. Takano K, Yamamoto M, Takahashi H, Shinomura Y, Imai K, Himi T. Clinicopathologic similarities between Mikulicz disease and Küttner tumor. Am J Otolaryngol 2010;31:429-34.

18. Hamano H, Kawa S, Horiuchi A, Unno H, Furuya N, Akamatsu T, Fukushima M, Nikaido T, Nakayama K, Usuda N, Kiyosawa K. High serum IgG4 concentrations in patients with sclerosing pancreatitis. N Engl J Med 2001;344:732-8.

19. Umehara H, Okazaki K, Masaki Y, Kawano M, Yamamoto M, Saeki T, Matsui S, Sumida T, Mimori T, Tanaka Y, Tsubota K, Yoshino T, Kawa S, Suzuki R, Takegami T, Tomosugi N, Kurose N, Ishigaki Y, Azumi A, Kojima M, Nakamura S, Inoue D. Research Program for Intractable Disease by Ministry of Health, Labor and Welfare (MHLW) Japan G4 team. A novel clinical entity, IgG4-related disease (IgG4RD): general concept and details. Mod Rheumatol 2012;22:1-14.

20. Kakudo K1, Li Y, Hirokawa M, Ozaki T. Diagnosis of Hashimoto's thyroiditis and IgG4-related sclerosing disease. Pathol Int 2011;61:175-83.

21. Yamamoto M, Takahashi H, Ohara M, Suzuki C, Naishiro Y, Yamamoto H, Shinomura Y, Imai K. A new conceptualization for Mikulicz's disease as an IgG4-related plasmacytic disease. Mod Rheumatol 2006;16:335-40.

22. Zen Y, Nakanuma Y. Pathogenesis of IgG4-related disease. Curr Opin Rheumatol 2011;23:114-8.

23. Hara S, Kawano M, Mizushima I, Yamada K, Fujita K, Matsumura M, Yamagishi M, Sato Y, Yamaguchi Y, Nakanuma Y, Nagata M. A condition closely mimicking IgG4-related disease despite the absence of serum IgG4 elevation and IgG4-positive plasma cell infiltration. Mod Rheumatol 2014;2:1-6.

24. Tanaka A, Moriyama M, Nakashima H, Miyake K, Hayashida JN, Maehara T, Shinozaki S, Kubo Y, Nakamura S. Th2 and regulatory immune reactions contribute to IgG4 production and the initiation of Mikulicz disease. Arthritis Rheum 2012;64:254-63.

25. Ito M, Naruke Y, Mihara Y, So K, Miyashita T, Origuchi T, Nakashima M, Livolsi V. Thyroid papillary carcinoma with solid sclerosing change in IgG4-related sclerosing disease. Pathol Int 2011;61:589-92.

26. Gill J, Angelo N, Yeong ML, McIvor N. Salivary duct carcinoma arising in IgG4-related autoimmune disease of the parotid gland. Hum Pathol 2009;40:881-6.

27. Taşli F, Ozkök G, Argon A, Ersöz D, Yağci A, Uslu A, Erkan N, Salman T, Vardar E. The role of IgG4 (+) plasma cells in the association of Hashimoto's thyroiditis with papillary carcinoma. APMIS 2014;122:1259-65.

28. Abe T, Sato T, Tomaru Y, Sakata Y, Kokabu S, Hori N, Kobayashi A, Yoda T. Immunoglobulin G4-related sclerosing sialadenitis: report of two cases and review of the literature. Oral Surg Oral Med Oral Pathol Oral Radiol 2009;108:544-50.

29. Doe K, Nozawa K, Okada T, Tada K, Yamaji K, Tamura N, Takasaki Y. Usefulness of minor salivary gland biopsy in the diagnosis of IgG4-related disease: a case report. Int J Clin Exp Pathol 2014;7:2673-7.

30. Gallo A, Martellucci S, Fusconi M, Pagliuca G, Greco A, De Virgilio A, de Vincentiis M. Sialendoscopic management of autoimmune sialadenitis: a review of literature. Acta Otorhinolaryngol Ital 2017;37:148-54.

31. Putra J, Ornstein DL. Küttner tumor: IgG4-related disease of the submandibular gland. Head Neck Pathol 2016;10:530-2.

32. Witte T, Schulze-Koops H. IgG4-related sialadenitis: IgG4 is helpful, but biopsies are still crucial. Arthritis Res Ther 2015;17:368. 